\title{
The Rough Guide to Constraint Propagation
}

\author{
Krzysztof R. Apt ${ }^{1,2}$ \\ 1 CWI \\ P.O. Box 94079, 1090 GB Amsterdam, the Netherlands \\ K.R.Apt@ewi.nl \\ 2 University of Amsterdam, the Netherlands
}

\begin{abstract}
We provide here a simple, yet very general framework that allows us to explain several constraint propagation algorithms in a systematic way. In particular, using the notions commutativity and semi-commutativity, we show how the well-known AC-3, PC-2, DAC and DPC algorithms are instances of a single generic algorithm. The work reported here extends and simplifies that of Apt [1].
\end{abstract}

\section{Introduction}

Constraint programming in a nutshell consists of formulating and solving so-called constraint satisfaction problems. One of the most important techniques developed in this area is constraint propagation that aims at reducing the search space while maintaining equivalence.

We call the corresponding algorithms constraint propagation algorithms but several other names have also been used in the literature: consistency, local consistency, consistency enforcing, Waltz, filtering or narrowing algorithms. These algorithms usually aim at reaching some form of "local consistency", a notion that in a loose sense approximates the notion of "global consistency".

Over the last twenty few years several constraint propagation algorithms were proposed and many of them are built into the existing constraint programming systems. In Apt [1] we introduced a simple framework that allows us to explain many of these algorithms in a uniform way. In this framework the notion of chaotic iterations, so fair iterations of functions, on Cartesian products of specific partial orderings played a crucial role. In Monfroy and Réty [13] this framework was modified to study distributed chaotic iterations. This resulted in a general framework for distributed constraint propagation algorithms.

We stated in Apt [1] that "the attempts of finding general principles behind the constraint propagation algorithms repeatedly reoccur in the literature on constraint satisfaction problems spanning the last twenty years" and devoted three pages to survey this work. Two references that are perhaps closest to our work are Benhamou [2] and Telerman and Ushakov [16].

These developments led to an identification of a number of mathematical properties that are of relevance for the considered functions, namely monotonicity, inflationarity and idempotence (see, e.g., Saraswat, Rinard and Panangaden [15] and Benhamou and 
Older [3]). Here we show that also the notions of commutativity and so-called semicommutativity are important.

As in Apt [1], to explain the constraint propagation algorithms, we proceed here in two steps. First, we introduce a generic iteration algorithm on partial orderings and prove its correctness in an abstract setting. Then we instantiate this algorithm with specific partial orderings and functions. The partial orderings will be related to the considered variable domains and the assumed constraints, while the functions will be the ones that characterize considered notions of local consistency in terms of fixpoints.

This presentation allows us to clarify which properties of the considered functions are responsible for specific properties of the corresponding algorithms. The resulting analysis is simpler than that of Apt [1] because we concentrate here on constraint propagation algorithms that always terminate. This allows us to dispense with the notion of fairness. On the other hand, we can now prove stronger results by taking into account the commutativity and semi-commutativity information.

This article is organized as follows. First, in Section 2, drawing on the approach of Monfroy and Réty [13], we introduce a generic algorithm for the case when the partial ordering is not further analyzed. Next, in Section 3, we refine it for the case when the partial ordering is a Cartesian product of component partial orderings and in Section 4 explain how the introduced notions should be related to the constraint satisfaction problems.

In the next four sections we instantiate the algorithm of Section 2 or some of its refinements to obtain specific constraint propagation algorithms. In particular, in Section 5 we derive algorithms for arc consistency and hyper-arc consistency. These algorithms can be improved by taking into account information on commutativity. This is done in Section 6 and yields the well-known AC-3 algorithm. Next, in Section 7 we derive an algorithm for path consistency and in Section 8 we improve it, again by using information on commutativity. This yields the $\mathrm{PC}-2$ algorithm.

In Section 9 we clarify under what assumptions the generic algorithm of Section 2 can be simplified to a simple for loop statement. Then we instantiate this simplified algorithm to derive in Section 10 the DAC algorithm for directional arc consistency and in Section 11 the DPC algorithm for directional path consistency. Finally, in Section 12 we briefly discuss possible future work.

So we deal here only with the classical algorithms that establish (directional) arc consistency and (directional) path consistency and that are more than twenty, respectively ten, years old. However, several more "modern" constraint propagation algorithms can also be explained in this framework. In particular, in Apt [1] page 203] we derived from a generic algorithm a simple algorithm that achieves the notion of relational consistency of Dechter and van Beek [7]. In turn, we can use the framework of Section 9 to derive the adaptive consistency algorithm of Dechter and Pearl [6]. Now, Dechter [5] showed that this algorithm can be formulated in a very general framework of bucket elimination that in turn can be used to explain such well-known algorithms as directional resolution, Fourier-Motzkin elimination, Gaussian elimination, and also various algorithms that deal with belief networks.

Due to lack of space we do not define here formally the considered local consistency notions and refer the interested reader instead to the original papers or to Tsang [17]. 


\section{Generic Iteration Algorithms}

Our presentation is completely general. Consequently, we delay the discussion of constraint satisfaction problems till Section . In what follows we shall rely on the following concepts.

Definition 1. Consider a partial ordering $(D, \sqsubseteq)$ with the least element $\perp$ and a finite set of functions $F:=\left\{f_{1}, \ldots, f_{k}\right\}$ on $D$.

- By an iteration of $F$ we mean an infinite sequence of values $d_{0}, d_{1}, \ldots$ defined inductively by

$$
\begin{gathered}
d_{0}:=\perp, \\
d_{j}:=f_{i_{j}}\left(d_{j-1}\right),
\end{gathered}
$$

where each $i_{j}$ is an element of $[1 . . k]$.

- We say that an increasing sequence $d_{0} \sqsubseteq d_{1} \sqsubseteq d_{2} \ldots$ of elements from $D$ eventually stabilizes at $\mathrm{d}$ iffor some $j \geq 0$ we have $d_{i}=d$ for $i \geq j$.

In what follows we shall consider iterations of functions that satisfy some specific properties.

Definition 2. Consider a partial ordering $(D, \sqsubseteq)$ and a function $f$ on $D$.

- $f$ is called inflationary if $x \sqsubseteq f(x)$ for all $x$.

- $f$ is called monotonic if $x \sqsubseteq y$ implies $f(x) \sqsubseteq f(y)$ for all $x, y$.

The following simple observation clarifies the role of monotonicity. The subsequent result will clarify the role of inflationarity.

Lemma 1 (Stabilization). Consider a partial ordering $(D, \sqsubseteq)$ with the least element $\perp$ and a finite set of monotonic functions $F$ on $D$.

Suppose that an iteration of $F$ eventually stabilizes at a common fixpoint $d$ of the functions from $F$. Then $d$ is the least common fixed point of the functions from $F$.

Proof. Consider a common fixpoint $e$ of the functions from $F$. We prove that $d \sqsubseteq e$. Let $d_{0}, d_{1}, \ldots$ be the iteration in question. For some $j \geq 0$ we have $d_{i}=d$ for $i \geq j$.

It suffices to prove by induction on $i$ that $d_{i} \sqsubseteq e$. The claim obviously holds for $i=0$ since $d_{0}=\perp$. Suppose it holds for some $i \geq 0$. We have $d_{i+1}=f_{j}\left(d_{i}\right)$ for some $j \in[1 . . k]$.

By the monotonicity of $f_{j}$ and the induction hypothesis we get $f_{j}\left(d_{i}\right) \sqsubseteq f_{j}(e)$, so $d_{i+1} \sqsubseteq e$ since $e$ is a fixpoint of $f_{j}$.

We fix now a partial ordering $(D, \sqsubseteq)$ with the least element $\perp$ and a set of functions $F:=\left\{f_{1}, \ldots, f_{k}\right\}$ on $D$. We are interested in computing the least common fixpoint of the functions from $F$. To this end we study the following algorithm that is inspired by a similar algorithm of Monfroy and Réty [13]. 


\section{GENERIC ITERATION ALgORITHM (GI)}

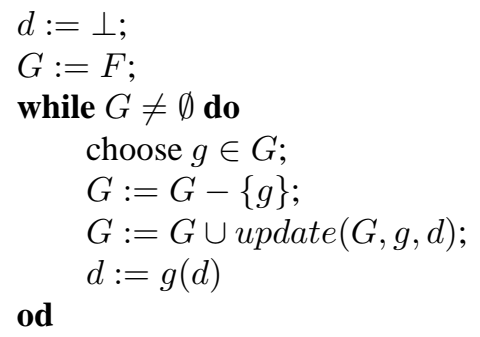

where for all $G, g, d$ the set of functions update $(G, g, d)$ from $F$ is such that

A. $\{f \in F-G \mid f(d)=d \wedge f(g(d)) \neq g(d)\} \subseteq$ update $(G, g, d)$,

B. $g(d)=d$ implies that update $(G, g, d)=\emptyset$.

Intuitively, assumption $\mathbf{A}$ states that update $(G, g, d)$ at least contains all the functions from $F-G$ for which $d$ is a fixpoint but $g(d)$ is not. The idea is that such functions are repeatedly added to the set $G$. In turn, assumption $\mathbf{B}$ states that no functions are added to $G$ in case the value of $d$ did not change.

An obvious example of an update function that satisfies assumptions $\mathbf{A}$ and $\mathbf{B}$ is

$$
\text { update }(G, g, d):=\{f \in F-G \mid f(d)=d \wedge f(g(d)) \neq g(d)\} .
$$

However, this choice of the update function is computationally expensive because for each function $f$ in $F-G$ we would have to compute the values $f(g(d))$ and $f(d)$. In practice, we are interested in some approximations of the above update function. We shall deal with this matter in the next section.

We now prove correctness of this algorithm in the following sense.

\section{Theorem 1 (GI) .}

(i) Every terminating execution of the GI algorithm computes in d a common fixpoint of the functions from $F$.

(ii) Suppose that all functions in $F$ are monotonic. Then every terminating execution of the GI algorithm computes in d the least common fixpoint of the functions from $F$.

(iii) Suppose that all functions in $F$ are inflationary and that $(D, \sqsubseteq)$ is finite. Then every execution of the $\mathrm{GI}$ algorithm terminates.

Proof.

(i) Consider the predicate $I$ defined by:

$$
I:=\forall f \in F-G f(d)=d .
$$

Note that $I$ is established by the assignment $G:=F$. Moreover, it is easy to check that $I$ is preserved by each while loop iteration. Thus $I$ is an invariant of the while loop of the algorithm. Hence upon its termination

$$
(G=\emptyset) \wedge I
$$


holds, that is

$$
\forall f \in F f(d)=d
$$

(ii) This is a direct consequence of $(i)$ and the Stabilization Lemma1.

( iii) Consider the lexicographic ordering of the partial orderings $(D, \sqsupseteq)$ and $(\mathcal{N}, \leq)$, defined on the elements of $D \times \mathcal{N}$ by

$$
\left(d_{1}, n_{1}\right) \leq_{\text {lex }}\left(d_{2}, n_{2}\right) \text { iff } d_{1} \sqsupset d_{2} \text { or }\left(d_{1}=d_{2} \text { and } n_{1} \leq n_{2}\right) .
$$

We use here the inverse ordering $\sqsupset$ defined by: $d_{1} \sqsupset d_{2}$ iff $d_{2} \sqsubseteq d_{1}$ and $d_{2} \neq d_{1}$.

Given a finite set $G$ we denote by $\operatorname{card} G$ the number of its elements. By assumption all functions in $F$ are inflationary so, by virtue of assumption $\mathbf{B}$, with each while loop iteration of the modified algorithm the pair

$$
(d, \operatorname{card} G)
$$

strictly decreases in this ordering $\leq_{l e x}$. But by assumption $(D, \sqsubseteq)$ is finite, so $(D, \sqsupseteq)$ is well-founded and consequently so is $\left(D \times \mathcal{N}, \leq_{\text {lex }}\right)$. This implies termination.

In particular, we obtain the following conclusion.

Corollary 1 (GI). Suppose that $(D, \sqsubseteq)$ is a finite partial ordering with the least element $\perp$. Let $F$ be a finite set of monotonic and inflationary functions on $D$. Then every execution of the GI algorithm terminates and computes in $d$ the least common fixpoint of the functions from $F$.

In practice, we are not only interested that the update function is easy to compute but also that it generates small sets of functions. Therefore we show how the function update can be made smaller when some additional information about the functions in $F$ is available. This will yield specialized versions of the GI algorithm. First we need the following simple concepts.

Definition 3. Consider two functions $f, g$ on a set $D$.

- We say that $f$ and $g$ commute if $f(g(x))=g(f(x))$ for all $x$.

- We call $f$ idempotent if $f(f(x))=f(x)$ for all $x$.

The following result holds.

\section{Theorem 2 (Update).}

(i) If update $(G, g, d)$ satisfies assumptions $\mathbf{A}$ and $\mathbf{B}$, then so does the function

$$
\text { update }(G, g, d)-\{g \mid g \text { is idempotent }\} \text {. }
$$

(ii) Suppose that for each $g \in F$ the set of functions Comm $(g)$ from $F$ is such that - $g \notin \operatorname{Comm}(g)$,

- each element of Comm $(g)$ commutes with $g$. 
If update $(G, g, d)$ satisfies assumptions $\mathbf{A}$ and $\mathbf{B}$, then so does the function

$$
\text { update }(G, g, d)-\operatorname{Comm}(g) \text {. }
$$

Proof. It suffices to establish in each case assumption $\mathbf{A}$.

(i) Suppose that $g$ is idempotent. Then any function $f$ such that $f(g(d)) \neq g(d)$ differs from $g$.

(ii) Consider a function $f$ from $F-G$ such that $f(d)=d$ and $f(g(d)) \neq g(d)$. Suppose that $f \in \operatorname{Comm}(g)$. Then $f(g(d))=g(f(d))=g(d)$ which is a contradiction. So $f \notin$ $\operatorname{Comm}(g)$. Consequently, $f \in$ update $(G, g, d)-\operatorname{Comm}(g)$ by virtue of assumption A for update $(G, g, d)$.

We conclude that given an instance of the GI algorithm that employs a specific update function, we can obtain other instances of it by using update functions modified as above. Note that both modifications are independent of each other and therefore can be applied together. In particular, when each function is idempotent and the function Comm satisfied the assumptions of $(i i)$, then if update $(G, g, d)$ satisfies assumptions $\mathbf{A}$ and $\mathbf{B}$, then so does the function update $(G, g, d)-(\operatorname{Comm}(g) \cup\{g\})$.

\section{Compound Domains}

In the applications we study the iterations are carried out on a partial ordering that is a Cartesian product of the partial orderings. So assume now that the partial ordering $(D, \sqsubseteq)$ is the Cartesian product of some partial orderings $\left(D_{i}, \sqsubseteq_{i}\right)$, for $i \in[1 . . n]$, each with the least element $\perp_{i}$. So $D=D_{1} \times \cdots \times D_{n}$.

Further, we assume that each function from $F$ depends from and affects only certain components of $D$. To be more precise we introduce a simple notation and terminology.

Definition 4. Consider a sequence of partial orderings $\left(D_{1}, \sqsubseteq_{1}\right), \ldots,\left(D_{n}, \sqsubseteq_{n}\right)$.

- By a scheme (on $n$ ) we mean a growing sequence of different elements from [1..n].

- Given a scheme $s:=i_{1}, \ldots, i_{l}$ on $n$ we denote by $\left(D_{s}, \sqsubseteq s\right)$ the Cartesian product of the partial orderings $\left(D_{i_{j}}, \sqsubseteq i_{j}\right)$, for $j \in[1 . . l]$.

- Given a function $f$ on $D_{s}$ we say that $f$ is with scheme $s$ and say that $f$ depends on $i$ if $i$ is an element of $s$.

- Given an $n$-tuple $d:=d_{1}, \ldots, d_{n}$ from $D$ and a scheme $s:=i_{1}, \ldots, i_{l}$ on $n$ we denote by $d[s]$ the tuple $d_{i_{1}}, \ldots, d_{i_{l}}$. In particular, for $j \in[1 . . n] d[j]$ is the $j$-th element of $d$.

Consider now a function $f$ with scheme $s$. We extend it to a function $f^{+}$from $D$ to $D$ as follows. Take $d \in D$. We set

$$
f^{+}(d):=e
$$

where $e[s]=f(d[s])$ and $e[n-s]=d[n-s]$, and where $n-s$ is the scheme obtained by removing from $1, \ldots, n$ the elements of $s$. We call $f^{+}$the canonic extension of $f$ to the domain $D$. 
So $f^{+}\left(d_{1}, \ldots, d_{n}\right)=\left(e_{1}, \ldots, e_{n}\right)$ implies $d_{i}=e_{i}$ for any $i$ not in the scheme $s$ of $f$. Informally, we can summarize it by saying that $f^{+}$does not change the components on which it does not depend. This is what we meant above by stating that each considered function affects only certain components of $D$.

We now say that two functions, $f$ with scheme $s$ and $g$ with scheme $t$ commute if the functions $f^{+}$and $g^{+}$commute.

Instead of defining iterations for the case of the functions with schemes, we rather reduce the situation to the one studied in the previous section and consider, equivalently, the iterations of the canonic extensions of these functions to the common domain $D$. However, because of this specific form of the considered functions, we can use now a simple definition of the update function. More precisely, we have the following observation.

Note 1 (Update). Suppose that each function in $F$ is of the form $f^{+}$. Then the following function update satisfies assumptions $\mathbf{A}$ and $\mathbf{B}$ :

$$
\begin{aligned}
& \text { update }\left(G, g^{+}, d\right):= \\
& \left\{f^{+} \in F-G \mid f \text { depends on some } i \text { in } s \text { such that } d[i] \neq g^{+}(d)[i]\right\},
\end{aligned}
$$

where $g$ is with scheme $s$.

Proof. To deal with assumption A take a function $f^{+} \in F-G$ such that $f^{+}(d)=d$. Then $f(e)=e$ for any $e$ that coincides with $d$ on all components that are in the scheme of $f$.

Suppose now additionally that $f^{+}\left(g^{+}(d)\right) \neq g^{+}(d)$. By the above $g^{+}(d)$ differs from $d$ on some component $i$ in the scheme of $f$. In other words, $f$ depends on some $i$ such that $d[i] \neq g^{+}(d)[i]$. This $i$ is then in the scheme of $g$.

The proof for assumption $\mathbf{B}$ is immediate.

This, together with the GI algorithm, yields the following algorithm in which we introduced a variable $d^{\prime}$ to hold the value of $g^{+}(d)$, and used $F_{0}:=\left\{f \mid f^{+} \in F\right\}$ and the functions with schemes instead of their canonic extensions to $D$.

\section{Generic Iteration Algorithm For COMPOUnd Domains (CD)}

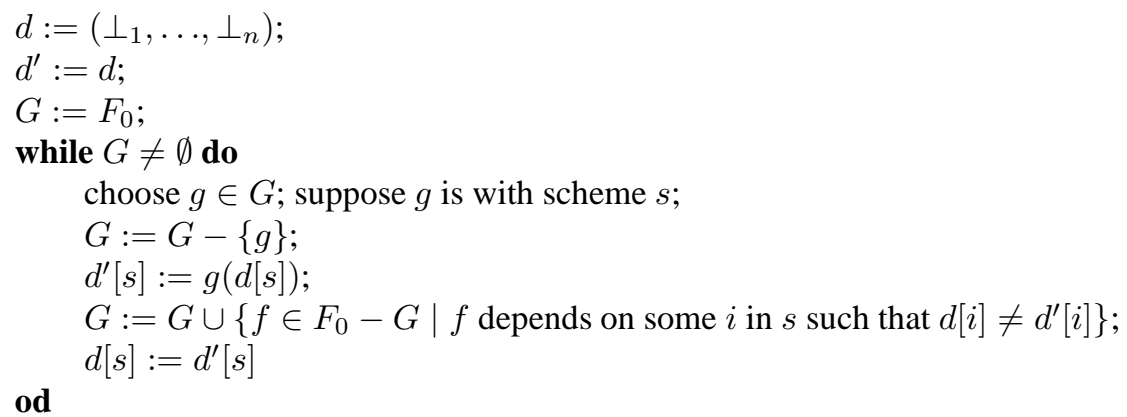

The following corollary to the GI Theorem 1 and the Update Note 11 summarizes the correctness of this algorithm. 
Corollary $2(\mathrm{CD})$. Suppose that $(D, \sqsubseteq)$ is a finite partial ordering that is a Cartesian product of $n$ partial orderings, each with the least element $\perp_{i}$ with $i \in[1 . . n]$. Let $F$ be a finite set of functions on $D$, each of the form $f^{+}$.

Suppose that all functions in $F$ are monotonic and inflationary. Then every execution of the $\mathrm{CD}$ algorithm terminates and computes in $d$ the least common fixpoint of the functions from $F$.

In the subsequent presentation we shall deal with the following two modifications of the $\mathrm{CD}$ algorithm:

- CDI algorithm. This is the version of the CD algorithm in which all the functions are idempotent and the function update defined in the Update Theorem $2(i)$ is used.

- $\mathrm{CDC}$ algorithm. This is the version of the $\mathrm{CD}$ algorithm in which all the functions are idempotent and the combined effect of the functions update defined in the Update Theorem 2 is used for some function Comm.

For both algorithms the counterparts of the CD Corollary 2 hold.

\section{From Partial Orderings to Constraint Satisfaction Problems}

We have been so far completely general in our discussion. Recall that our aim is to derive various constraint propagation algorithms. To be able to apply the results of the previous section we need to relate various abstract notions that we used there to constraint satisfaction problems.

This is perhaps the right place to recall the definition and to fix the notation. Consider a finite sequence of variables $X:=x_{1}, \ldots, x_{n}$, where $n \geq 0$, with respective domains $\mathcal{D}:=D_{1}, \ldots, D_{n}$ associated with them. So each variable $x_{i}$ ranges over the domain $D_{i}$. By a constraint $C$ on $X$ we mean a subset of $D_{1} \times \ldots \times D_{n}$.

By a constraint satisfaction problem, in short CSP, we mean a finite sequence of variables $X$ with respective domains $\mathcal{D}$, together with a finite set $\mathcal{C}$ of constraints, each on a subsequence of $X$. We write it as $\left\langle\mathcal{C} ; x_{1} \in D_{1}, \ldots, x_{n} \in D_{n}\right\rangle$, where $X:=$ $x_{1}, \ldots, x_{n}$ and $\mathcal{D}:=D_{1}, \ldots, D_{n}$.

Consider now an element $d:=d_{1}, \ldots, d_{n}$ of $D_{1} \times \ldots \times D_{n}$ and a subsequence $Y:=x_{i_{1}}, \ldots, x_{i_{\ell}}$ of $X$. Then we denote by $d[Y]$ the sequence $d_{i_{1}}, \ldots, d_{i_{\ell}}$.

By a solution to $\left\langle\mathcal{C} ; x_{1} \in D_{1}, \ldots, x_{n} \in D_{n}\right\rangle$ we mean an element $d \in D_{1} \times$ $\ldots \times D_{n}$ such that for each constraint $C \in \mathcal{C}$ on a sequence of variables $Y$ we have $d[Y] \in C$. We call a CSP consistent if it has a solution. Two CSP's $\mathcal{P}_{1}$ and $\mathcal{P}_{2}$ with the same sequence of variables are called equivalent if they have the same set of solutions. This definition extends in an obvious way to the case of two CSP's with the same sets of variables.

Let us return now to the framework of the previous section. It involved:

(i) Partial orderings with the least elements;

These will correspond to partial orderings on the CSP's. In each of them the original CSP will be the least element and the partial ordering will be determined by the local consistency notion we wish to achieve. 
(ii) Monotonic and inflationary functions with schemes;

These will correspond to the functions that transform the variable domains or the constraints. Each function will be associated with one or more constraints.

(iii) Common fixpoints;

These will correspond to the CSP's that satisfy the considered notion of local consistency.

In what follows we shall discuss two specific partial orderings on the CSP's. In each of them the considered CSP's will be defined on the same sequences of variables.

We begin by fixing for each set $D$ a collection $\mathcal{F}(D)$ of the subsets of $D$ that includes $D$ itself. So $\mathcal{F}$ is a function that given a set $D$ yields a set of its subsets to which $D$ belongs.

When dealing with the hyper-arc consistency $\mathcal{F}(D)$ will be simply the set $\mathcal{P}(D)$ of all subsets of $D$ but for specific domains only specific subsets of $D$ will be chosen. For example, to deal with the the constraint propagation for the linear constraints on integer interval domains we need to choose for $\mathcal{F}(D)$ the set of all subintervals of the original interval $D$.

When dealing with the path consistency, for a constraint $C$ the collection $\mathcal{F}(C)$ will be also the set $\mathcal{P}(C)$ of all subsets of $C$. However, in general other choices may be needed. For example, to deal with the cutting planes method, we need to limit our attention to the sets of integer solutions to finite sets of linear inequalities with integer coefficients (see Apt [1, pages 193-194]).

Next, given two CSP's, $\phi:=\left\langle\mathcal{C} ; x_{1} \in D_{1}, \ldots, x_{n} \in D_{n}\right\rangle$ and $\psi:=\left\langle\mathcal{C}^{\prime} ; x_{1} \in\right.$ $\left.D_{1}^{\prime}, \ldots, x_{n} \in D_{n}^{\prime}\right\rangle$, we write $\phi \sqsubseteq_{d} \psi$ iff

- $D_{i}^{\prime} \in \mathcal{F}\left(D_{i}\right)$ (and hence $\left.D_{i}^{\prime} \subseteq D_{i}\right)$ for $i \in[1 . . n]$,

- the constraints in $\mathcal{C}^{\prime}$ are the restrictions of the constraints in $\mathcal{C}$ to the domains $D_{1}^{\prime}, \ldots, D_{n}^{\prime}$.

So $\phi \sqsubseteq_{d} \psi$ if $\psi$ can be obtained from $\phi$ by a domain reduction rule and the domains of $\psi$ belong to the appropriate collections of sets $\mathcal{F}(D)$.

Next, given two CSP's, $\phi:=\left\langle C_{1}, \ldots, C_{k} ; \mathcal{D E}\right\rangle$ and $\psi:=\left\langle C_{1}^{\prime}, \ldots, C_{k}^{\prime} ; \mathcal{D E}\right\rangle$, we write $\phi \sqsubseteq_{c} \psi$ iff

- $C_{i}^{\prime} \in \mathcal{F}\left(C_{i}\right)$ (and hence $C_{i}^{\prime} \subseteq C_{i}$ ) for $i \in[1 . . k]$.

In what follows we call $\sqsubseteq_{d}$ the domain reduction ordering and $\sqsubseteq_{c}$ the constraint reduction ordering. To deal with the arc consistency, hyper-arc consistency and directional arc consistency notions we shall use the domain reduction ordering, and to deal with path consistency and directional path consistency notions we shall use the constraint reduction ordering.

We consider each ordering with some fixed initial CSP $\mathcal{P}$ as the least element. In other words, each domain reduction ordering is of the form

$$
\left(\left\{\mathcal{P}^{\prime} \mid \mathcal{P} \sqsubseteq_{d} \mathcal{P}^{\prime}\right\}, \sqsubseteq_{d}\right)
$$

and each constraint reduction ordering is of the form

$$
\left(\left\{\mathcal{P}^{\prime} \mid \mathcal{P} \sqsubseteq_{c} \mathcal{P}^{\prime}\right\}, \sqsubseteq_{c}\right) .
$$


Note that $\left\langle\mathcal{C} ; x_{1} \in D_{1}^{\prime}, \ldots, x_{n} \in D_{n}^{\prime}\right\rangle \sqsubseteq_{d}\left\langle\mathcal{C}^{\prime} ; x_{1} \in D_{1}^{\prime \prime}, \ldots, x_{n} \in D_{n}^{\prime \prime}\right\rangle$ iff $D_{i}^{\prime} \supseteq D_{i}^{\prime \prime}$ for $i \in[1 . . n]$.

This means that for $\mathcal{P}=\left\langle\mathcal{C} ; x_{1} \in D_{1}, \ldots, x_{n} \in D_{n}\right\rangle$ we can identify the domain reduction ordering $\left(\left\{\mathcal{P}^{\prime} \mid \mathcal{P} \sqsubseteq_{d} \mathcal{P}^{\prime}\right\}, \sqsubseteq_{d}\right)$ with the Cartesian product of the partial orderings $\left(\mathcal{F}\left(D_{i}\right), \supseteq\right)$, where $i \in[1 . . n]$. Additionally, each CSP in this domain reduction ordering is uniquely determined by its domains and by the initial $\mathcal{P}$.

Similarly,

$$
\left\langle C_{1}^{\prime}, \ldots, C_{k}^{\prime} ; \mathcal{D E}\right\rangle \sqsubseteq_{c}\left\langle C_{1}^{\prime \prime}, \ldots, C_{k}^{\prime \prime} ; \mathcal{D} \mathcal{E}\right\rangle \text { iff } C_{i}^{\prime} \supseteq C_{i}^{\prime \prime} \text { for } i \in[1 . . k] .
$$

This allows us for $\mathcal{P}=\left\langle C_{1}, \ldots, C_{k} ; \mathcal{D E}\right\rangle$ to identify the constraint reduction ordering $\left(\left\{\mathcal{P}^{\prime} \mid \mathcal{P} \sqsubseteq_{c} \mathcal{P}^{\prime}\right\}, \sqsubseteq_{c}\right)$ with the Cartesian product of the partial orderings $\left(\mathcal{F}\left(C_{i}\right), \supseteq\right.$ ) , where $i \in[1 . . k]$. Also, each CSP in this constraint reduction ordering is uniquely determined by its constraints and by the initial $\mathcal{P}$.

In what follows instead of the domain reduction ordering and the constraint reduction ordering we shall use the corresponding Cartesian products of the partial orderings. So in these compound orderings the sequences of the domains (respectively, of the constraints) are ordered componentwise by the reversed subset ordering $\supseteq$. Further, in each component ordering $(\mathcal{F}(D), \supseteq)$ the set $D$ is the least element.

Consider now a function $f$ on some Cartesian product $\mathcal{F}\left(E_{1}\right) \times \ldots \times \mathcal{F}\left(E_{m}\right)$. Note that $f$ is inflationary w.r.t. the componentwise ordering $\supseteq$ if for all $\left(X_{1}, \ldots, X_{m}\right) \in$ $\mathcal{F}\left(E_{1}\right) \times \ldots \times \mathcal{F}\left(E_{m}\right)$ we have $Y_{i} \subseteq X_{i}$ for all $i \in[1 . . m]$, where $f\left(X_{1}, \ldots, X_{m}\right)=$ $\left(Y_{1}, \ldots, Y_{m}\right)$.

Also, $f$ is monotonic w.r.t. the componentwise ordering $\supseteq$ if for all $\left(X_{1}, \ldots, X_{m}\right)$, $\left(X_{1}^{\prime}, \ldots, X_{m}^{\prime}\right) \in \mathcal{F}\left(E_{1}\right) \times \ldots \times \mathcal{F}\left(E_{m}\right)$ such that $X_{i} \subseteq X_{i}^{\prime}$ for all $i \in[1 . . m]$, the following holds: if

$$
f\left(X_{1}, \ldots, X_{m}\right)=\left(Y_{1}, \ldots, Y_{m}\right) \text { and } f\left(X_{1}^{\prime}, \ldots, X_{m}^{\prime}\right)=\left(Y_{1}^{\prime}, \ldots, Y_{m}^{\prime}\right),
$$

then $Y_{i} \subseteq Y_{i}^{\prime}$ for all $i \in[1 . . m]$.

In other words, $f$ is monotonic w.r.t. $\supseteq$ iff it is monotonic w.r.t. $\subseteq$. This reversal of the set inclusion of course does not hold for the inflationarity notion.

\section{A Hyper-arc Consistency Algorithm}

We begin by considering the notion of hyper-arc consistency of Mohr and Masini [12] (we use here the terminology of Marriott and Stuckey [10]). The more known notion of arc consistency of Mackworth [9] is obtained by restricting one's attention to binary constraints.

To employ the CDI algorithm of Section 3 we now make specific choices involving the items (i), (ii) and (iii) of the previous section.

Re: (i) Partial orderings with the least elements.

As already mentioned in the previous section, for the function $\mathcal{F}$ we choose the powerset function $\mathcal{P}$, so for each domain $D$ we put $\mathcal{F}(D):=\mathcal{P}(D)$.

Given a CSP $\mathcal{P}$ with the sequence $D_{1}, \ldots, D_{n}$ of the domains we take the domain reduction ordering with $\mathcal{P}$ as its least element. As already noted we can identify this 
ordering with the Cartesian product of the partial orderings $\left(\mathcal{P}\left(D_{i}\right), \supseteq\right)$, where $i \in$ $[1 . . n]$. The elements of this compound ordering are thus sequences $\left(X_{1}, \ldots, X_{n}\right)$ of respective subsets of the domains $D_{1}, \ldots, D_{n}$ ordered componentwise by the reversed subset ordering $\supseteq$.

Re: (ii) Monotonic and inflationary functions with schemes.

Given a constraint $C$ on the variables $y_{1}, \ldots, y_{k}$ with respective domains $E_{1}, \ldots, E_{k}$, we abbreviate for each $j \in[1 . . k]$ the set $\{d[j] \mid d \in C\}$ to $\Pi_{j}(C)$. Thus $\Pi_{j}(C)$ consists of all $j$-th coordinates of the elements of $C$. Consequently, $\Pi_{j}(C)$ is a subset of the domain $E_{j}$ of the variable $y_{j}$.

We now introduce for each $i \in[1 . . k]$ the following function $\pi_{i}$ on $\mathcal{P}\left(E_{1}\right) \times \cdots \times$ $\mathcal{P}\left(E_{k}\right)$ :

$$
\pi_{i}\left(X_{1}, \ldots, X_{k}\right):=\left(X_{1}, \ldots, X_{i-1}, X_{i}^{\prime}, X_{i+1}, \ldots, X_{k}\right)
$$

where

$$
X_{i}^{\prime}:=\Pi_{i}\left(C \cap\left(X_{1} \times \cdots \times X_{k}\right)\right) .
$$

That is, $X_{i}^{\prime}=\left\{d[i] \mid d \in X_{1} \times \cdots \times X_{k}\right.$ and $\left.d \in C\right\}$. Each function $\pi_{i}$ is associated with a specific constraint $C$. Note that $X_{i}^{\prime} \subseteq X_{i}$, so each function $\pi_{i}$ boils down to a projection on the $i$-th component.

Re: (iii) Common fixpoints.

Their use is clarified by the following lemma that also lists the relevant properties of the functions $\pi_{i}$.

Lemma 2 (Hyper-arc Consistency).

(i) $A \operatorname{CSP}\left\langle\mathcal{C} ; x_{1} \in D_{1}, \ldots, x_{n} \in D_{n}\right\rangle$ is hyper-arc consistent iff $\left(D_{1}, \ldots, D_{n}\right)$ is a common fixpoint of all functions $\pi_{i}^{+}$associated with the constraints from $\mathcal{C}$.

(ii) Each projection function $\pi_{i}$ associated with a constraint $C$ is

- inflationary w.r.t. the componentwise ordering $\supseteq$,

- monotonic w.r.t. the componentwise ordering $\supseteq$,

- idempotent.

By taking into account only the binary constraints we obtain an analogous characterization of arc consistency. The functions $\pi_{1}$ and $\pi_{2}$ can then be defined more directly as follows:

$$
\pi_{1}(X, Y):=\left(X^{\prime}, Y\right)
$$

where $X^{\prime}:=\{a \in X \mid \exists b \in Y(a, b) \in C\}$, and

$$
\pi_{2}(X, Y):=\left(X, Y^{\prime}\right)
$$

where $Y^{\prime}:=\{b \in Y \mid \exists a \in X(a, b) \in C\}$.

Fix now a CSP $\mathcal{P}$. By instantiating the $C D I$ algorithm with

$$
F_{0}:=\left\{f \mid f \text { is a } \pi_{i} \text { function associated with a constraint of } \mathcal{P}\right\}
$$

and with each $\perp_{i}$ equal to $D_{i}$ we get the HYPER-ARC algorithm that enjoys following properties. 
Theorem 3 (HYPER-ARC Algorithm). Consider a CSP $\mathcal{P}:=\left\langle\mathcal{C} ; x_{1} \in D_{1}, \ldots, x_{n} \in\right.$ $\left.D_{n}\right\rangle$ where each $D_{i}$ is finite.

The HYPER-ARC algorithm always terminates. Let $\mathcal{P}^{\prime}$ be the CSP determined by $\mathcal{P}$ and the sequence of the domains $D_{1}^{\prime}, \ldots, D_{n}^{\prime}$ computed in $d$. Then

(i) $\mathcal{P}^{\prime}$ is the $\sqsubseteq_{d}$-least CSP that is hyper-arc consistent,

(ii) $\mathcal{P}^{\prime}$ is equivalent to $\mathcal{P}$.

Due to the definition of the $\sqsubseteq_{d}$ ordering the item $(i)$ can be rephrased as follows. Consider all hyper-arc consistent CSP's that are of the form $\left\langle\mathcal{C}^{\prime} ; x_{1} \in D_{1}^{\prime}, \ldots, x_{n} \in\right.$ $\left.D_{n}^{\prime}\right\rangle$ where $D_{i}^{\prime} \subseteq D_{i}$ for $i \in[1 . . n]$ and the constraints in $\mathcal{C}^{\prime}$ are the restrictions of the constraints in $\mathcal{C}$ to the domains $D_{1}^{\prime}, \ldots, D_{n}^{\prime}$. Then among these CSP's $\mathcal{P}^{\prime}$ has the largest domains.

\section{An Improvement: the $A C-3$ Algorithm}

In this section we show how we can exploit an information about the commutativity of the $\pi_{i}$ functions. Recall that in Section 3 we modified the notion of commutativity for the case of functions with schemes. We now need the following lemma.

Lemma 3 (Commutativity). Consider a CSP and two constraints of it, $C$ on the variables $y_{1}, \ldots, y_{k}$ and $E$ on the variables $z_{1}, \ldots, z_{\ell}$.

(i) For $i, j \in[1 . . k]$ the functions $\pi_{i}$ and $\pi_{j}$ of the constraint $C$ commute.

(ii) If the variables $y_{i}$ and $z_{j}$ are identical then the functions $\pi_{i}$ of $C$ and $\pi_{j}$ of $E$ commute.

Fix now a CSP. We derive a modification of the HYPER-ARC algorithm by instantiating this time the CDC algorithm. As before we use the set of functions $F_{0}:=$ $\left\{f \mid f\right.$ is a $\pi_{i}$ function associated with a constraint of $\left.\mathcal{P}\right\}$ and each $\perp_{i}$ equal to $D_{i}$. Additionally we employ the following function Comm, where $\pi_{i}$ is associated with a constraint $C$ :

$$
\begin{aligned}
\operatorname{Comm}\left(\pi_{i}\right):= & \left\{\pi_{j} \mid i \neq j \text { and } \pi_{j} \text { is associated with the constraint } C\right\} \\
& \cup\left\{\pi_{j} \mid \pi_{j} \text { is associated with a constraint } E\right. \text { and } \\
& \text { the } i \text {-th variable of } C \text { and the } j \text {-th variable of } E \text { coincide }\} .
\end{aligned}
$$

By virtue of the Commutativity Lemma 3 each set $\operatorname{Comm}(g)$ satisfies the assumptions of the Update Theorem $2($ ii $)$.

By limiting oneself to the set of functions $\pi_{1}$ and $\pi_{2}$ associated with the binary constraints, we obtain an analogous modification of the corresponding arc consistency algorithm.

Using now the counterpart of the $C D$ Corollary 2 for the $C D C$ algorithm we conclude that the above algorithm enjoys the same properties as the HYPER-ARC algorithm, that is the counterpart of the HYPER-ARC Algorithm Theorem 3 holds.

Let us clarify now the difference between this algorithm and the HYPER-ARC algorithm when both of them are limited to the binary constraints. 
Assume that the considered CSP is of the form $\langle\mathcal{C} ; \mathcal{D E}\rangle$. We reformulate the above algorithm as follows. Given a binary relation $R$, we put

$$
R^{T}:=\{(b, a) \mid(a, b) \in R\} .
$$

For $F_{0}$ we now choose the set of the $\pi_{1}$ functions of the constraints or relations from the set

$$
S_{0}:=\{C \mid C \text { is a binary constraint from } \mathcal{C}\}
$$

$\cup\left\{C^{T} \mid C\right.$ is a binary constraint from $\left.\mathcal{C}\right\}$.

Finally, for each $\pi_{1}$ function of some $C \in S_{0}$ on $x, y$ we define

$$
\begin{aligned}
\operatorname{Comm}\left(\pi_{1}\right) & :=\left\{f \mid f \text { is the } \pi_{1} \text { function of } C^{T}\right\} \\
& \cup\left\{f \mid f \text { is the } \pi_{1} \text { function of some } E \in S_{0} \text { on } x, z \text { where } z \not \equiv y\right\} .
\end{aligned}
$$

Assume now that

$$
\text { for each pair of variables } x, y \text { at most one constraint exists on } x, y \text {. }
$$

Consider now the corresponding instance of the CDC algorithm. By incorporating into it the effect of the functions $\pi_{1}$ on the corresponding domains, we obtain the following algorithm known as the $\mathrm{AC}-3$ algorithm of Mackworth [9].

We assume here that $\mathcal{D E}:=x_{1} \in D_{1}, \ldots, x_{n} \in D_{n}$.

AC-3 Algorithm

$S_{0}:=\{C \mid C$ is a binary constraint from $\mathcal{C}\}$

$\cup\left\{C^{T} \mid C\right.$ is a binary constraint from $\left.\mathcal{C}\right\}$;

$S:=S_{0}$

while $S \neq \emptyset$ do

choose $C \in S$; suppose $C$ is on $x_{i}, x_{j}$;

$D_{i}:=\left\{a \in D_{i} \mid \exists b \in D_{j}(a, b) \in C\right\}$;

if $D_{i}$ changed then

fi;

$S:=S \cup\left\{C^{\prime} \in S_{0} \mid C^{\prime}\right.$ is on the variables $y, x_{i}$ where $\left.y \not \equiv x_{j}\right\}$

$S:=S-\{C\}$

od

It is useful to mention that the corresponding reformulation of the HYPER-ARC algorithm differs in the second assignment to $S$ which is then

$$
S:=S \cup\left\{C^{\prime} \in S_{0} \mid C^{\prime} \text { is on the variables } y, z \text { where } y \text { is } x_{i} \text { or } z \text { is } x_{i}\right\} .
$$

So we "capitalized" here on the commutativity of the corresponding projection functions $\pi_{1}$ as follows. First, no constraint or relation on $x_{i}, z$ for some $z$ is added to $S$. Here we exploited part $(i i)$ of the Commutativity Lemma 3 .

Second, no constraint or relation on $x_{j}, x_{i}$ is added to $S$. Here we exploited part $(i)$ of the Commutativity Lemma 3 , because by assumption (1) $C^{T}$ is the only constraint or relation on $x_{j}, x_{i}$ and its $\pi_{1}$ function coincides with the $\pi_{2}$ function of $C$. 
In case the assumption (11) about the considered CSP is dropped, the resulting algorithm is somewhat less readable. However, once we use the following modified definition of $\operatorname{Comm}\left(\pi_{1}\right)$ :

$$
\operatorname{Comm}\left(\pi_{1}\right):=\left\{f \mid f \text { is the } \pi_{1} \text { function of some } E \in S_{0} \text { on } x, z \text { where } z \not \equiv y\right\}
$$

we get an instance of the $\mathrm{CDC}$ algorithm which differs from the $\mathrm{AC}-3$ algorithm in that the qualification "where $y \not \equiv x_{j}$ " is removed from the definition of the second assignment to the set $S$.

\section{A Path Consistency Algorithm}

The notion of path consistency was introduced in Montanari [14]. It is defined for special type of CSP's. For simplicity we ignore here unary constraints that are usually present when studying path consistency.

Definition 5. We call a CSP normalized if it has only binary constraints and for each pair $x$, y of its variables exactly one constraint on them exists. We denote this constraint by $C_{x, y}$.

Every CSP with only unary and binary constraints is trivially equivalent to a normalized CSP. Consider now a normalized CSP $\mathcal{P}$. Suppose that $\mathcal{P}=\left\langle C_{1}, \ldots, C_{k} ; \mathcal{D E}\right\rangle$.

We proceed now as in the case of hyper-arc consistency. First, we choose for the function $\mathcal{F}$ the powerset function. For the partial ordering we choose the constraint reduction ordering of Section $\sharp$, or rather its counterpart which is the Cartesian product of the partial orderings $\left(\mathcal{P}\left(C_{i}\right), \supseteq\right)$, where $i \in[1 . . k]$.

Second, we introduce appropriate monotonic and inflationary functions with schemes. To this end, given two binary relations $R$ and $S$ we define their composition - by

$$
R \cdot S:=\{(a, b) \mid \exists c((a, c) \in R,(c, b) \in S)\} .
$$

Note that if $R$ is a constraint on the variables $x, y$ and $S$ a constraint on the variables $y, z$, then $R \cdot S$ is a constraint on the variables $x, z$.

Given a subsequence $x, y, z$ of the variables of $\mathcal{P}$ we now introduce three functions on $\mathcal{P}\left(C_{x, y}\right) \times \mathcal{P}\left(C_{x, z}\right) \times \mathcal{P}\left(C_{y, z}\right)$ :

$$
f_{x, y}^{z}(P, Q, R):=\left(P^{\prime}, Q, R\right)
$$

where $P^{\prime}:=P \cap Q \cdot R^{T}$,

$$
f_{x, z}^{y}(P, Q, R):=\left(P, Q^{\prime}, R\right),
$$

where $Q^{\prime}:=Q \cap P \cdot R$, and

$$
f_{y, z}^{x}(P, Q, R):=\left(P, Q, R^{\prime}\right),
$$

where $R^{\prime}:=R \cap P^{T} \cdot Q$.

Finally, we introduce common fixpoints of the above defined functions. To this end we need the following counterpart of the Hyper-arc Consistency Lemma 2 . 


\section{Lemma 4 (Path Consistency).}

(i) A normalized $\operatorname{CSP}\left\langle C_{1}, \ldots, C_{k} ; \mathcal{D E}\right\rangle$ is path consistent iff $\left(C_{1}, \ldots, C_{k}\right)$ is a common fixpoint of all functions $\left(f_{x, y}^{z}\right)^{+},\left(f_{x, z}^{y}\right)^{+}$and $\left(f_{y, z}^{x}\right)^{+}$associated with the subsequences $x, y, z$ of its variables.

(ii) The functions $f_{x, y}^{z}, f_{x, z}^{y}$ and $f_{y, z}^{x}$ are

- inflationary w.r.t. the componentwise ordering $\supseteq$,

- monotonic w.r.t. the componentwise ordering ?,

- idempotent.

We now instantiate the CDI algorithm with the set of functions

$F_{0}:=\left\{f \mid x, y, z\right.$ is a subsequence of the variables of $\mathcal{P}$ and $\left.f \in\left\{f_{x, y}^{z}, f_{x, z}^{y}, f_{y, z}^{x}\right\}\right\}$,

$n:=k$ and each $\perp_{i}$ equal to $C_{i}$.

Call the resulting algorithm the PATH algorithm. It enjoys the following properties.

Theorem 4 (PATH Algorithm). Consider a normalized CSP $\mathcal{P}:=\left\langle C_{1}, \ldots, C_{k} ; \mathcal{D E}\right\rangle$. Assume that each constraint $C_{i}$ is finite.

The РАTH algorithm always terminates. Let $\mathcal{P}^{\prime}:=\left\langle C_{1}^{\prime}, \ldots, C_{k}^{\prime} ; \mathcal{D E}\right\rangle$, where the sequence of the constraints $C_{1}^{\prime}, \ldots, C_{k}^{\prime}$ is computed in $d$. Then

(i) $\mathcal{P}^{\prime}$ is the $\sqsubseteq_{c}$-least CSP that is path consistent,

(ii) $\mathcal{P}^{\prime}$ is equivalent to $\mathcal{P}$.

As in the case of the HYPER-ARC Algorithm Theorem 3 the item $(i)$ can be rephrased as follows. Consider all path consistent CSP's that are of the form $\left\langle C_{1}^{\prime}, \ldots, C_{k}^{\prime} ; \mathcal{D E}\right\rangle$ where $C_{i}^{\prime} \subseteq C_{i}$ for $i \in[1 . . k]$. Then among them $\mathcal{P}^{\prime}$ has the largest constraints.

\section{An Improvement: the $\mathrm{PC}-2$ Algorithm}

As in the case of the hyper-arc consistency we can improve the PATH algorithm by taking into account the commutativity information.

Fix a normalized CSP $\mathcal{P}$. We abbreviate the statement " $x, y$ is a subsequence of the variables of $\mathcal{P}$ " to $x \prec y$. We now have the following lemma.

Lemma 5 (Commutativity). Suppose that $x \prec y$ and let $z, u$ be some variables of $\mathcal{P}$ such that $\{u, z\} \cap\{x, y\}=\emptyset$. Then the functions $f_{x, y}^{z}$ and $f_{x, y}^{u}$ commute.

In other words, two functions with the same pair of variables as a subscript commute.

We now instantiate the CDC algorithm with the same set of functions $F_{0}$ as in Section 7 . Additionally, we use the function Comm defined as follows, where $x \prec y$ and where $z \notin\{x, y\}$ :

$$
\operatorname{Comm}\left(f_{x, y}^{z}\right)=\left\{f_{x, y}^{u} \mid u \notin\{x, y, z\}\right\} .
$$

Thus for each function $g$ the set $\operatorname{Comm}(g)$ contains precisely $m-3$ elements, where $m$ is the number of variables of the considered CSP. This quantifies the maximal 
"gain" obtained by using the commutativity information: at each "update" stage of the corresponding instance of the CDC algorithm we add up to $m-3$ less elements than in the case of the corresponding instance of the CDI algorithm considered in the previous section.

By virtue of the Commutativity Lemma 5 each set $\operatorname{Comm}(g)$ satisfies the assumptions of the Update Theorem 2(ii). We conclude that the above instance of the CDC algorithm enjoys the same properties as the original PATH algorithm, that is the counterpart of the PATH Algorithm Theorem 4 holds. To make this modification of the PATH algorithm easier to understand we proceed as follows.

Each function of the form $f_{x, y}^{u}$ where $x \prec y$ and $u \notin\{x, y\}$ can be identified with the sequence $x, u, y$ of the variables. (Note that the "relative" position of $u$ w.r.t. $x$ and $y$ is not fixed, so $x, u, y$ does not have to be a subsequence of the variables of $\mathcal{P}$.) This allows us to identify the set of functions $F_{0}$ with the set

$$
V_{0}:=\{(x, u, y) \mid x \prec y, u \notin\{x, y\}\} .
$$

Next, assuming that $x \prec y$, we introduce the following set of triples of different variables of $\mathcal{P}$ :

$$
\begin{aligned}
V_{x, y} & :=\{(x, y, u) \mid x \prec u\} \cup\{(y, x, u) \mid y \prec u\} \\
& \cup\{(u, x, y) \mid u \prec y\} \cup\{(u, y, x) \mid u \prec x\} .
\end{aligned}
$$

Informally, $V_{x, y}$ is the subset of $V_{0}$ that consists of the triples that begin or end with either $x, y$ or $y, x$. This corresponds to the set of functions in one of the following forms: $f_{x, u}^{y}, f_{y, u}^{x}, f_{u, y}^{x}$ and $f_{u, x}^{y}$.

The above instance of the $\mathrm{CDC}$ algorithm then becomes the following PC-2 algorithm of Mackworth [9]. Here initially $E_{x, y}=C_{x, y}$.

PC-2 Algorithm

$V_{0}:=\{(x, u, y) \mid x \prec y, u \notin\{x, y\}\}$

$V:=V_{0}$;

while $V \neq \emptyset$ do

choose $p \in V$; suppose $p=(x, u, y)$;

apply $f_{x, y}^{u}$ to its current domains;

if $E_{x, y}$ changed then

fi;

$V:=V \cup V_{x, y}$;

od

$V:=V-\{p\}$

Here the phrase "apply $f_{x, y}^{u}$ to its current domains" can be made more precise if the "relative" position of $u$ w.r.t. $x$ and $y$ is known. Suppose for instance that $u$ is "before" $x$ and $y$. Then $f_{x, y}^{u}$ is defined on $\mathcal{P}\left(C_{u, x}\right) \times \mathcal{P}\left(C_{u, y}\right) \times \mathcal{P}\left(C_{x, y}\right)$ by

$$
f_{x, y}^{u}\left(E_{u, x}, E_{u, y}, E_{x, y}\right):=\left(E_{u, x}, E_{u, y}, E_{x, y} \cap E_{u, x}^{T} \cdot E_{u, y}\right),
$$


so the above phrase "apply $f_{x, y}^{u}$ to its current domains" can be replaced by the assignment

$$
E_{x, y}:=E_{x, y} \cap E_{u, x}^{T} \cdot E_{u, y} .
$$

Analogously for the other two possibilities.

The difference between the $\mathrm{PC}-2$ algorithm and the corresponding representation of the PATH algorithm lies in the way the modification of the set $V$ is carried out. In the case of the PATH algorithm the second assignment to $V$ is

$$
V:=V \cup V_{x, y} \cup\{(x, u, y) \mid u \notin\{x, y\}\} .
$$

\section{Simple Iteration Algorithms}

Let us return now to the framework of Section 2 . We analyze here when the while loop of the Generic Iteration Algorithm Gi can be replaced by a for loop. First, we weaken the notion of commutativity as follows.

Definition 6. Consider a partial ordering $(D, \sqsubseteq)$ and functions $f$ and $g$ on $D$. We say that $f$ semi-commutes with $g$ (w.r.t. $\sqsubseteq)$ if $f(g(x)) \sqsubseteq g(f(x))$ for all $x$.

The following lemma provides an answer to the question just posed. Here and elsewhere we omit brackets when writing repeated applications of functions to an argument.

Lemma 6 (Simple Iteration). Consider a partial ordering $(D, \sqsubseteq)$ with the least element $\perp$. Let $F:=f_{1}, \ldots, f_{k}$ be a finite sequence of monotonic, inflationary and idempotent functions on $D$. Suppose that $f_{i}$ semi-commutes with $f_{j}$ for $i>j$, that is,

$$
f_{i}\left(f_{j}(x)\right) \sqsubseteq f_{j}\left(f_{i}(x)\right) \text { for all } x \text {. }
$$

Then $f_{1} f_{2} \ldots f_{k}(\perp)$ is the least common fixpoint of the functions from $F$.

Proof. We prove first that for $i \in[1 . . k]$ we have

$$
f_{i} f_{1} f_{2} \ldots f_{k}(\perp) \sqsubseteq f_{1} f_{2} \ldots f_{k}(\perp) .
$$

Indeed, by the assumption (2) we have the following string of inclusions, where the last one is due to the idempotence of the considered functions:

$$
f_{i} f_{1} f_{2} \ldots f_{k}(\perp) \sqsubseteq f_{1} f_{i} f_{2} \ldots f_{k}(\perp) \sqsubseteq \ldots \sqsubseteq f_{1} f_{2} \ldots f_{i} f_{i} \ldots f_{k}(\perp) \sqsubseteq f_{1} f_{2} \ldots f_{k}(\perp) .
$$

Additionally, by the inflationarity of the considered functions, we also have for $i \in$ $[1 . . k]$

$$
f_{1} f_{2} \ldots f_{k}(\perp) \sqsubseteq f_{i} f_{1} f_{2} \ldots f_{k}(\perp) .
$$

So $f_{1} f_{2} \ldots f_{k}(\perp)$ is a common fixpoint of the functions from $F$. This means that the iteration of $F$ that starts with $\perp, f_{k}(\perp), f_{k-1} f_{k}(\perp), \ldots, f_{1} f_{2} \ldots f_{k}(\perp)$ eventually stabilizes at $f_{1} f_{2} \ldots f_{k}(\perp)$. By the Stabilization Lemma 1 we get the desired conclusion. 
The above lemma provides us with a simple way of computing the least common fixpoint of a set of finite functions that satisfy the assumptions of this lemma, in particular condition (2). Namely, it suffices to order these functions in an appropriate way and then to apply each of them just once, starting with the argument $\perp$.

To this end we maintain the considered functions not in a set but in a list. Given a non-empty list $L$ we denote its head by $\operatorname{head}(L)$ and its tail by $\operatorname{tail}(L)$. Next, given a sequence of elements $a_{1}, \ldots, a_{n}$ with $n \geq 0$, we denote by $\left[a_{1}, \ldots, a_{n}\right]$ the list formed by them. If $n=0$, then this list is empty and is denoted by [] and if $n>0$, then $\operatorname{head}\left(\left[a_{1}, \ldots, a_{n}\right]\right)=a_{1}$ and tail $\left(\left[a_{1}, \ldots, a_{n}\right]\right)=\left[a_{2}, \ldots a_{n}\right]$.

The following algorithm is a counterpart of the GI algorithm. We assume in it that condition (2) holds for the functions $f_{1}, \ldots, f_{k}$.

Simple Iteration Algorithm (SI)

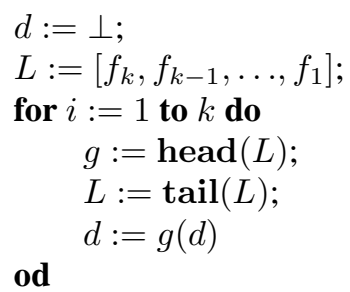

The following immediate consequence of the Simple Iteration Lemma 6 is a counterpart of the GI Corollary 1 .

Corollary 3 (SI) . Suppose that $(D, \sqsubseteq)$ is a partial ordering with the least element $\perp$. Let $F:=f_{1}, \ldots, f_{k}$ be a finite sequence of monotonic, inflationary and idempotent functions on D such that (2) holds. Then the SI algorithm terminates and computes in $d$ the least common fixpoint of the functions from $F$.

Note that in contrast to the GI Corollary 1 we do not require here that the partial ordering is finite. Because at each iteration of the for loop exactly one element is removed from the list $L$, at the end of this loop the list $L$ is empty. Consequently, this algorithm is a reformulation of the one in which the line

$$
\text { for } i:=1 \text { to } k \text { do }
$$

is replaced by

$$
\text { while } L \neq \text { [ ] do. }
$$

So we can view the SI algorithm as a specialization of the GI algorithm of Section 2 in which the elements of the set of functions $G$ (here represented by the list $L$ ) are selected in a specific way and in which the update function always yields the empty set.

In Section 3 we refined the GI algorithm for the case of compound domains. An analogous refinement of the SI algorithm is straightforward and omitted. In the next two sections we show how we can use this refinement of the SI algorithm to derive two well-known constraint propagation algorithms. 


\section{DAC: a Directional Arc Consistency Algorithm}

We consider here the notion of directional arc consistency of Dechter and Pearl [6]. To derive an algorithm that achieves this local consistency notion we first characterize it in terms of fixpoints. To this end, given a $\mathcal{P}$ and a linear ordering $\prec$ on its variables, we rather reason in terms of the equivalent CSP $\mathcal{P} \prec$ obtained from $\mathcal{P}$ by reordering its variables along $\prec$ so that each constraint in $\mathcal{P} \prec$ is on a sequence of variables $x_{1}, \ldots, x_{k}$ such that $x_{1} \prec x_{2} \prec \ldots \prec x_{k}$.

The following characterization holds.

Lemma 7 (Directional Arc Consistency). Consider a CSP $\mathcal{P}$ with a linear ordering $\prec$ on its variables. Let $\mathcal{P}_{\prec}:=\left\langle\mathcal{C} ; x_{1} \in D_{1}, \ldots, x_{n} \in D_{n}\right\rangle$. Then $\mathcal{P}$ is directionally arc consistent w.r.t. $\prec$ iff $\left(D_{1}, \ldots, D_{n}\right)$ is a common fixpoint of the functions $\pi_{1}^{+}$associated with the binary constraints from $\mathcal{P}_{\prec}$.

We now instantiate in an appropriate way the SI algorithm for compound domains with all the $\pi_{1}$ functions associated with the binary constraints from $\mathcal{P}_{\prec}$. In this way we obtain an algorithm that achieves for $\mathcal{P}$ directional arc consistency w.r.t. $\prec$. First, we adjust the definition of semi-commutativity to functions with different schemes. To this end consider a sequence of partial orderings $\left(D_{1}, \sqsubseteq_{1}\right), \ldots,\left(D_{n}, \sqsubseteq_{n}\right)$ and their Cartesian product $(D, \sqsubseteq)$. Take two functions, $f$ with scheme $s$ and $g$ with scheme $t$. We say that $f$ semi-commutes with $g$ (w.r.t. $\sqsubseteq$ ) if $f^{+}$semi-commutes with $g^{+}$w.r.t. $\sqsubseteq$, that is if

$$
f^{+}\left(g^{+}(Q)\right) \sqsubseteq g^{+}\left(f^{+}(Q)\right)
$$

for all $Q \in D$.

The following lemma is crucial.

Lemma 8 (Semi-commutativity). Consider a CSP and two binary constraints of it, $C_{1}$ on $u, z$ and $C_{2}$ on $x, y$, where $y \prec z$.

Then the $\pi_{1}$ function of $C_{1}$ semi-commutes with the $\pi_{1}$ function of $C_{2}$ w.r.t. the componentwise ordering $\supseteq$.

Consider now a CSP $\mathcal{P}$ with a linear ordering $\prec$ on its variables and the corresponding CSP $\mathcal{P}_{\prec}$. To be able to apply the above lemma we order the $\pi_{1}$ functions of the binary constraints of $\mathcal{P}_{\prec}$ in an appropriate way. Namely, given two $\pi_{1}$ functions, $f$ associated with a constraint on $u, z$ and $g$ associated with a constraint on $x, y$, we put $f$ before $g$ if $y \prec z$.

More precisely, let $x_{1}, \ldots, x_{n}$ be the sequence of the variables of $\mathcal{P} \prec$. So $x_{1} \prec x_{2} \prec$ $\ldots \prec x_{n}$. Let for $m \in[1 . . n]$ the list $L_{m}$ consist of the $\pi_{1}$ functions of those binary constraints of $\mathcal{P}_{\prec}$ that are on $x_{j}, x_{m}$ for some $x_{j}$. We order each list $L_{m}$ arbitrarily. Consider now the list $L$ resulting from appending $L_{n}, L_{n-1}, \ldots, L_{1}$, in that order, so with the elements of $L_{n}$ in front. Then by virtue of the Semi-commutativity Lemma 8 if the function $f$ precedes the function $g$ in the list $L$, then $f$ semi-commutes with $g$ w.r.t. the componentwise ordering $\supseteq$.

We instantiate now the refinement of the SI algorithm for the compound domains by the above-defined list $L$ and each $\perp_{i}$ equal to the domain $D_{i}$ of the variable $x_{i}$. We assume that $L$ has $k$ elements. We obtain then the following algorithm. 


\section{Directional ARC Consistency Algorithm (DARC)}

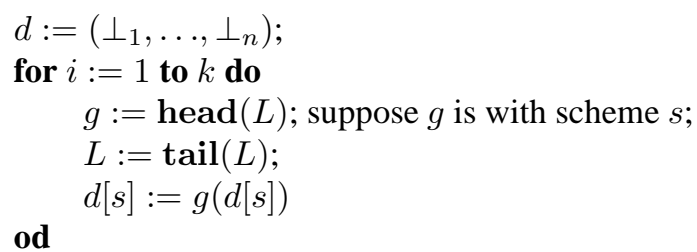

This algorithm enjoys the following properties.

Theorem 5 (DARC Algorithm). Consider a CSP $\mathcal{P}$ with a linear ordering $\prec$ on its variables. Let $\mathcal{P}_{\prec}:=\left\langle\mathcal{C} ; x_{1} \in D_{1}, \ldots, x_{n} \in D_{n}\right\rangle$.

The DARC algorithm always terminates. Let $\mathcal{P}^{\prime}$ be the CSP determined by $\mathcal{P} \prec$ and the sequence of the domains $D_{1}^{\prime}, \ldots, D_{n}^{\prime}$ computed in $d$. Then

(i) $\mathcal{P}^{\prime}$ is the $\sqsubseteq_{d}$-least CSP in $\left\{\mathcal{P}_{1} \mid \mathcal{P}_{\prec} \sqsubseteq_{d} \mathcal{P}_{1}\right\}$ that is directionally arc consistent w.r.t. $\prec$,

(ii) $\mathcal{P}^{\prime}$ is equivalent to $\mathcal{P}$.

Note that in contrast to the HYPER-ARC Algorithm Theorem 3 we do not need to assume here that each domain is finite.

Assume now that for each pair of variables $x, y$ of the original CSP $\mathcal{P}$ there exists precisely one constraint on $x, y$. The same holds then for $\mathcal{P}_{\prec}$. Suppose that $\mathcal{P}_{\prec}:=$ $\left\langle\mathcal{C} ; x_{1} \in D_{1}, \ldots, x_{n} \in D_{n}\right\rangle$. Denote the unique constraint of $\mathcal{P}_{\prec}$ on $x_{i}, x_{j}$ by $C_{i, j}$. The above DARC algorithm can then be rewritten as the following algorithm known as the DAC algorithm of Dechter and Pearl [6]:

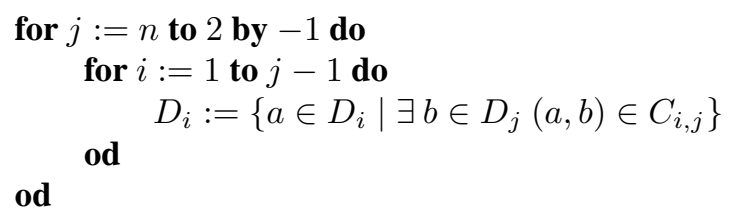

\section{DPC: a Directional Path Consistency Algorithm}

In this section we deal with the notion of directional path consistency defined in Dechter and Pearl [6]. As before we first characterize this local consistency notion in terms of fixpoints. To this end, as in the previous section, given a normalized CSP $\mathcal{P}$ we rather consider the equivalent CSP $\mathcal{P}_{\prec}$. The variables of $\mathcal{P}_{\prec}$ are ordered according to $\prec$ and on each pair of its variables there exists a unique constraint.

The following is a counterpart of the Directional Arc Consistency Lemma 7.

Lemma 9 (Directional Path Consistency). Consider a normalized CSP $\mathcal{P}$ with a linear ordering $\prec$ on its variables. Let $\mathcal{P}_{\prec}:=\left\langle C_{1}, \ldots, C_{k} ; \mathcal{D E}\right\rangle$. Then $\mathcal{P}$ is directionally path consistent w.r.t. $\prec$ iff $\left(C_{1}, \ldots, C_{k}\right)$ is a common fixpoint of all functions $\left(f_{x, y}^{z}\right)^{+}$ associated with the subsequences $x, y, z$ of the variables of $\mathcal{P}_{\prec}$. 
To obtain an algorithm that achieves directional path consistency we now instantiate in an appropriate way the SI algorithm. To this end we need the following lemma.

Lemma 10 (Semi-commutativity). Consider a normalized CSP and two subsequences of its variables, $x_{1}, y_{1}, z$ and $x_{2}, y_{2}, u$. Suppose that $u \prec z$.

Then the function $f_{x_{1}, y_{1}}^{z}$ semi-commutes with the function $f_{x_{2}, y_{2}}^{u}$ w.r.t. the componentwise ordering $\supseteq$.

Consider now a normalized CSP $\mathcal{P}$ with a linear ordering $\prec$ on its variables and the corresponding CSP $\mathcal{P}_{\prec}$. To be able to apply the above lemma we order in an appropriate way the $f_{r, s}^{t}$ functions, where the variables $r, s, t$ are such that $r \prec s \prec t$. Namely, we put $f_{x_{1}, y_{1}}^{z}$ before $f_{x_{2}, y_{2}}^{u}$ if $u \prec z$.

More precisely, let $x_{1}, \ldots, x_{n}$ be the sequence of the variables of $\mathcal{P}_{\prec}$, that is $x_{1} \prec$ $x_{2} \prec \ldots \prec x_{n}$. Let for $m \in[1 . . n]$ the list $L_{m}$ consist of the functions $f_{x_{i}, x_{j}}^{x_{m}}$ for some $x_{i}$ and $x_{j}$. We order each list $L_{m}$ arbitrarily and consider the list $L$ resulting from appending $L_{n}, L_{n-1}, \ldots, L_{1}$, in that order. Then by virtue of the Semi-commutativity Lemma 9 if the function $f$ precedes the function $g$ in the list $L$, then $f$ semi-commutes with $g$ w.r.t. the componentwise ordering $\supseteq$.

We instantiate now the refinement of the SI algorithm for the compound domains by the above-defined list $L$ and each $\perp_{i}$ equal to the constraint $C_{i}$. We assume that $L$ has $k$ elements. This yields the Directional Path Consistency Algorithm (DPATH) that, apart from of the different choice of the constituent partial orderings, is identical to the Directional ARC CONSISTENCY Algorithm DARC of the previous section. Consequently, the DPATH algorithm enjoys analogous properties as the DARC algorithm. They are summarized in the following theorem.

Theorem 6 (DPATH Algorithm). Consider a CSP $\mathcal{P}$ with a linear ordering $\prec$ on its variables. Let $\mathcal{P}_{\prec}:=\left\langle C_{1}, \ldots, C_{k} ; \mathcal{D E}\right\rangle$.

The DPATH algorithm always terminates. Let $\mathcal{P}^{\prime}:=\left\langle C_{1}^{\prime}, \ldots, C_{k}^{\prime} ; \mathcal{D E}\right\rangle$, where the sequence of the constraints $C_{1}^{\prime}, \ldots, C_{k}^{\prime}$ is computed in $d$. Then

(i) $\mathcal{P}^{\prime}$ is the $\sqsubseteq_{c}$-least CSP in $\left\{\mathcal{P}_{1} \mid \mathcal{P}_{\prec} \sqsubseteq_{d} \mathcal{P}_{1}\right\}$ that is directionally path consistent w.r.t. $\prec$

(ii) $\mathcal{P}^{\prime}$ is equivalent to $\mathcal{P}$.

As in the case of the DARC Algorithm Theorem 5 we do not need to assume here that each domain is finite.

Assume now that that $x_{1}, \ldots, x_{n}$ is the sequence of the variables of $\mathcal{P}_{\prec}$. Denote the unique constraint of $\mathcal{P}_{\prec}$ on $x_{i}, x_{j}$ by $C_{i, j}$.

The above DPATH algorithm can then be rewritten as the following algorithm known as the DPC algorithm of Dechter and Pearl [6]:

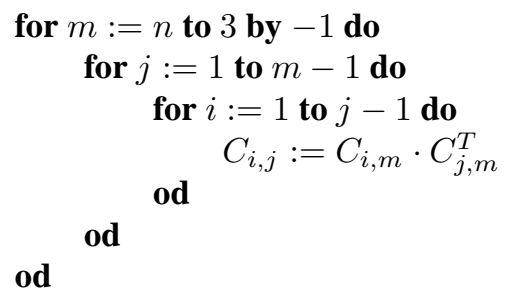




\section{Conclusions}

In this article we introduced a general framework for constraint propagation. It allowed us to present and explain various constraint propagation algorithms in a uniform way. Using such a single framework we can easier verify, compare, modify, parallelize or combine these algorithms. The last point has already been made to large extent in Benhamou [2]. Additionally, we clarified the role played by the notions of commutativity and semi-commutativity.

The line of research presented here could be extended in a number of ways. First, it would be interesting to find examples of existing constraint propagation algorithms that could be improved by using the notions of commutativity and semi-commutativity.

Second, as already stated in Apt [1], it would be useful to explain in a similar way other constraint propagation algorithms such as the AC-4 algorithm of Mohr and Henderson [11], the PC-4 algorithm of Han and Lee [8], or the GAC-4 algorithm of Mohr and Masini [12]. The complication is that these algorithms operate on some extension of the original CSP.

Finally, it would be useful to apply the approach of this paper to derive constraint propagation algorithms for the semiring-based constraint satisfaction framework of Bistarelli, Montanari and Rossi [4] that provides a unified model for several classes of "nonstandard" constraints satisfaction problems.

\section{References}

1. K. R. Apt. The essence of constraint propagation. Theoretical Computer Science, 221(12):179-210, 1999. Available via http://xxx.lanl.gov/archive/cs/.

2. F. Benhamou. Heterogeneous constraint solving. In M. Hanus and M. Rodriguez-Artalejo, editors, Proceeding of the Fifth International Conference on Algebraic and Logic Programming (ALP 96), Lecture Notes in Computer Science 1139, pages 62-76, Berlin, 1996. Springer-Verlag.

3. F. Benhamou and W. Older. Applying interval arithmetic to real, integer and Boolean constraints. Journal of Logic Programming, 32(1):1-24, 1997.

4. S. Bistarelli, U. Montanari, and F. Rossi. Semiring-based constraint satisfaction and optimization. Journal of the ACM, 44(2):201-236, March 1997.

5. R. Dechter. Bucket elimination: A unifying framework for structure-driven inference. Artificial Intelligence, 1999. To appear.

6. R. Dechter and J. Pearl. Network-based heuristics for constraint-satisfaction problems. Artificial Intelligence, 34(1):1-38, January 1988.

7. R. Dechter and P. van Beek. Local and global relational consistency. Theoretical Computer Science, 173(1):283-308, 20 February 1997.

8. C. Han and C. Lee. Comments on Mohr and Henderson's path consistency algorithm. Artificial Intelligence, 36:125-130, 1988.

9. A. Mackworth. Consistency in networks of relations. Artificial Intelligence, 8(1):99-118, 1977.

10. K. Marriott and P. Stuckey. Programming with Constraints. The MIT Press, Cambridge, Massachusetts, 1998.

11. R. Mohr and T.C. Henderson. Arc-consistency and path-consistency revisited. Artificial Intelligence, 28:225-233, 1986. 
12. R. Mohr and G. Masini. Good old discrete relaxation. In Y. Kodratoff, editor, Proceedings of the 8th European Conference on Artificial Intelligence (ECAI), pages 651-656. Pitman Publishers, 1988.

13. E. Monfroy and J.-H. Réty. Chaotic iteration for distributed constraint propagation. In J. Carroll, H. Haddad, D. Oppenheim, B. Bryant, and G. Lamont, editors, Proceedings of The 1999 ACM Symposium on Applied Computing, SAC'99, pages 19-24, San Antonio, Texas, USA, March 1999. ACM Press.

14. U. Montanari. Networks of constraints: Fundamental properties and applications to picture processing. Information Science, 7(2):95-132, 1974. Also Technical Report, Carnegie Mellon University, 1971.

15. V.A. Saraswat, M. Rinard, and P. Panangaden. Semantic foundations of concurrent constraint programming. In Proceedings of the Eighteenth Annual ACM Symposium on Principles of Programming Languages (POPL'91), pages 333-352, 1991.

16. V. Telerman and D. Ushakov. Data types in subdefinite models. In J. A. Campbell J. Calmet and J. Pfalzgraf, editors, Artificial Intelligence and Symbolic Mathematical Computations, Lecture Notes in Computer Science 1138, pages 305-319, Berlin, 1996. Springer-Verlag.

17. E. Tsang. Foundations of Constraint Satisfaction. Academic Press, 1993. 